\title{
Measuring tiny mass accretion rates onto young brown dwarfs
}

\author{
Gregory J. Herczeg, Kelle Cruz and Lynne A. Hillenbrand \\ Caltech, MC105-24, 1200 E. California Blvd., Pasadena, CA 91125
}

\begin{abstract}
We present low-resolution Keck I/LRIS-ADC spectra spanning from 3200-9000 ̊ of six young brown dwarfs in the TW Hya Association and in Upper Sco. The optical spectral types of the sample range from M7.25-M8.75, though two have near-IR spectral types of early L-dwarfs. For four brown dwarfs in our sample, the accretion luminosity is measurable only when the image is binned over large wavelength intervals. This method extends our sensivity to accretion rate down to $\sim 10^{-13} M_{\odot} \mathrm{yr}^{-1}$ for brown dwarfs.
\end{abstract}

Keywords: stars: pre-main sequence - stars: low-mass

PACS: $95.85 . \mathrm{Ls}, 97.10 . \mathrm{Gz}, 97.10 . \mathrm{Nf}$

\section{INTRODUCTION}

That young brown dwarfs share many characteristics with T Tauri stars is strong evidence that the formation and early evolution of stars and brown dwarfs is similar. However, the smaller masses of brown dwarfs introduce physical differences that alter the early evolution of the star+disk system. Protoplanetary disks around very low-mass stars and brown dwarfs survive longer than around solar-mass stars [2, 3, 15], which may relate to the $\dot{M} \propto M^{2}$ relationship that describes the average accretion rate for a population of objects with a large range of masses [e.g., 12].

Accretion rate measurements contribute to our understanding of viscous dissipation and photoevaporation of the disk. Accretion but is most accurately measured from excess Balmer continuum at $<3646 \AA$ [16, 4, 5]. Such UV-excess accretion rates range from $\dot{M} \sim 10^{-9}-10^{-7} \dot{M} \mathrm{yr}^{-1}$ for $0.5-1 M_{\odot}$ stars but can be $<10^{-12} \dot{M} \mathrm{yr}^{-1}$ for the lowest mass brown dwarfs. Sensitivity to small accretion rates is determined by the ability to measure the contrast between the star and hot photospheric emission, with cooler targets having smaller upper limits because of weaker and redder photospheric emission.

In these proceedings, we develop a method to extract very low-resolution spectra of the photospheric and accretion continuum from Keck I/LRIS optical spectra of faint sources. This technique is applied to a small sample of four brown dwarfs in the TWA and USco to measure accretion rates down to $10^{-13} M_{\odot} \mathrm{yr}^{-1}$, an order of magnitude lower than previous measurements.

\section{OBSERVATIONS}

We used LRIS-ADC [13, 9, 14] on Keck I to obtain $R \sim 1000$ spectra from 3000-9000 $\AA$ of the five brown dwarfs 2MASS J12073346-3932540, USco J160603.75-221930.0, 

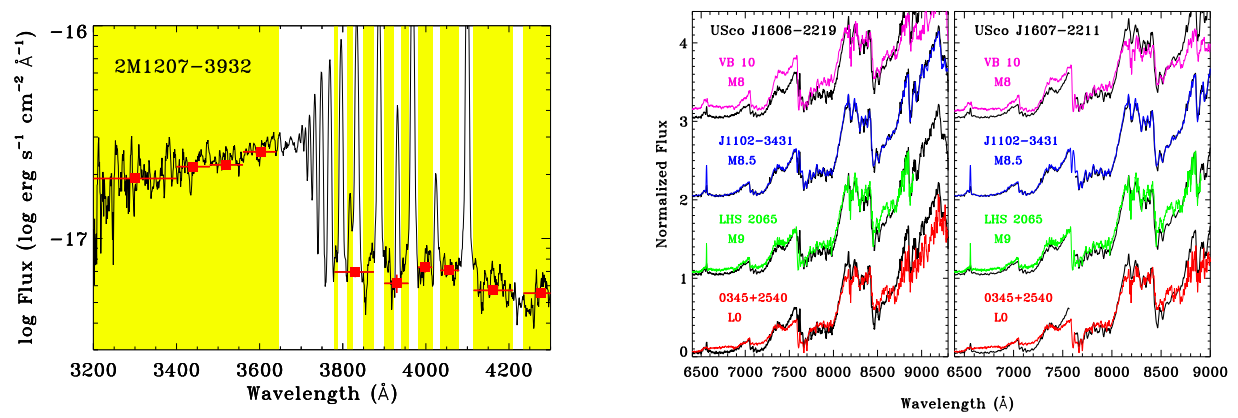

FIGURE 1. Left: Demonstrating our continuum measurements on our spectrum of 2MASS J120733463932540, which are applied to fainter targets in Figs. 2-3. The black is the reduced spectrum. The yellow shaded areas indicate regions where the continuum emission dominates over line emission. The red squares are the continuum emission measurements obtained from the binned image. Right: Red spectra of USco J160723.82-221102.0 (left) and USco J160603.75-221930.0 (right) are classified as young brown dwarfs with spectral types M8.5 and M8.75, respectively.

SSSPM J1102-3431, Oph J162225-240515A, and Oph J162225-240515B. We also obtained a red spectrum (5600-9000 ̊) of the brown dwarf USco J160723.82-221102.0. The continuum emission at 3200-4500 ̊ from USco J160603.75-221930.0, SSSPM J1102-3431, Oph J162225-240515A, and Oph J162225-240515B is too faint for simple long-slit extraction. Very low-resolution spectra of the continuum emission are extracted for these sources after binning the image along the dispersion direction. This method significantly reduces the noise introduced when calculating the background subtraction independently at each pixel in the dispersion direction. The image is binned over regions dominated by continuum emission to minimize contamination of our continuum flux measurements by strong lines. Our observation of 2MASS J12073347-3932540 is used to demonstrate this method (Fig. 1) because it is brighter than our other targets.

\section{SPECTRAL AND ACCRETION PROPERTIES}

The spectrum from 3200-9000 ̊̊ of a young brown dwarf consists of a red photospheric continuum and weak chromospheric emission in the Balmer and Ca II H \& $\mathrm{K}$ lines. Based on the depth of $\mathrm{VO}$ and $\mathrm{TiO}$ absorption bands, the five brown dwarfs in our sample range from M7.25-M8.75, which corresponds to masses of $0.019-0.055 M_{\odot}$. USco J160603.75-221930.0 and USco J160723.82-221102.0 had been previously identified as L2 and L1 brown dwarfs, respectively, based on low-resolution near-IR spectra [7]. This discrepancy is similar to that for young stars in IC348 [8]. The Luhman et al. [8] relationship between temperature and spectral type applies to optical spectral types, but no similar relation has been derived for near-IR spectral types. We caution against the use of near-IR spectral types to obtain temperatures and masses of young brown dwarfs until these effects are better characterized.

The presence of accretion alters the spectrum by producing excess emission in lines and $\mathrm{H}$ continua, including the so-called Balmer jump in emission at $\lambda<3646$ due to the Balmer continuum. Figure 2 shows the binned spectra of four faint targets compared with 

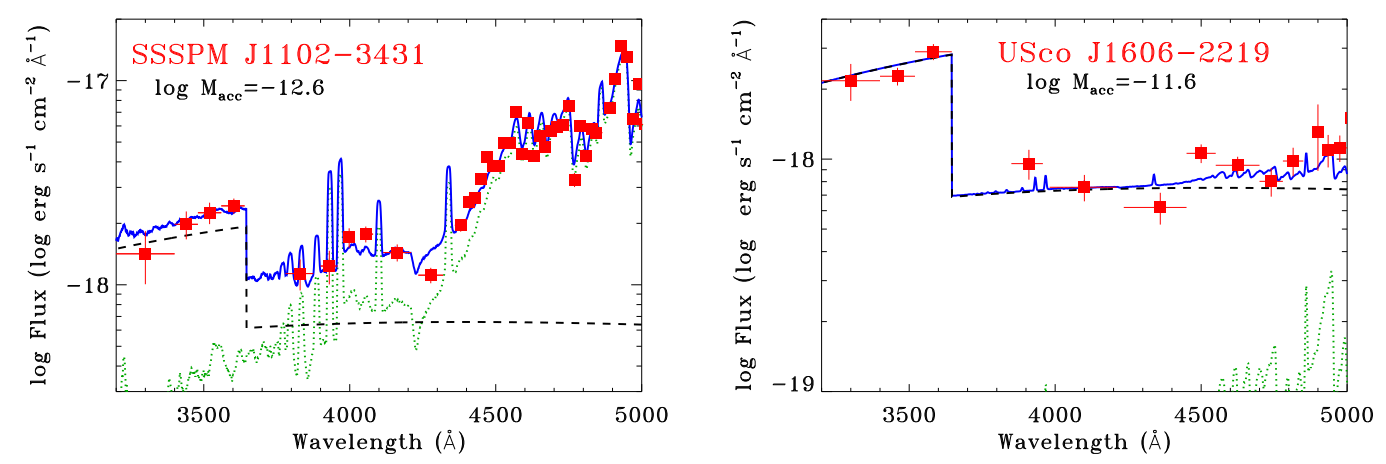

FIGURE 2. Continuum spectrophotometry of four faint young brown dwarfs (red squares). The dashed green line shows the spectrum of the photospheric template LHS 3003, scaled to the photospheric emission. The solid blue line shows the spectrum of LHS3003 plus the model accretion continuum (dashed black line). Excess emission is detected from both SSSPM J1102-3431 and USco J160603.75-221930.0. The spectrum of USco J160603.75-221930.0 is heavily veiled at $<5000 \AA$, while the veiling of SSSPM J1102-3431 is small longward of the Balmer jump at $3646 \AA$.

a photospheric template, the active M7 star LHS 3003. The blue continuum from SSSPM J1102-3431, Oph J162225-240515B, and Oph J162225-240515A falls rapidly toward shorter wavelenths, similar to the photospheric template. Any veiling due to accretion is undetected at $>4000 \AA$ in these three objects. In contrast, USco J160603.75-221930.0 has a relatively flat spectrum from $4000-5000 \AA$, consistent with high veiling.

SSSPM J1102-3431, Oph J162225-240515B, and USco J160603.75-221930.0 have excess emission at $<3700 \AA$, which is attributed to the Balmer continuum. The observed Balmer jumps $\left(B J_{o b s}\right.$ in Table 2$)$ for these three targets are larger than the observed Balmer jump of 0.35-0.45 for chromospherically-active M-dwarfs [5]. We classify these three stars as accretors based on excess Balmer continuum emission. The Balmer jump of $<0.4$ for USco J160723.82-221102.0 is lower than expected from a late-M brown dwarf photosphere and suggests that the star is not accreting.

The excess accretion continuum emission described above is produced near where the accreting gas shocks at the brown dwarf surface [1]. With a bolometric correction, the observed excess flux over a narrow wavelength range can be converted into a total accretion continuum luminosity, $L_{a c c}$ [4]. The accretion continuum is calculated by modelling a pure hydrogen, isothermal, plane-parallel slab [16, 5]. These models are simplistic and less physical than the shock models [1] but provide similar bolometric corrections and consistency with most previous accretion rate measurements. The accretion rates for SSSPM J1102-3431, USco J160603.75-221930.0, Oph J162225-240515B and an upper limit for Oph J162225-240515A are listed in Figs 2 and 3.

The sensitivity of UV-excess measurements to small accretion luminosities relies on the ability to detect the Balmer continuum in excess of the photospheric emission. At earlier spectral types, this limit is determined by the strength of the photospheric emission and an upper limit on the veiling, typically $\sim 0.1-0.3$, yielding upper limits of $10^{-9}-10^{-11} M_{\odot} \mathrm{yr}^{-1}$. Towards later spectral types, the fainter and redder photospheric emission becomes undetectable at short wavelengths. The telescopic sensitivity then 

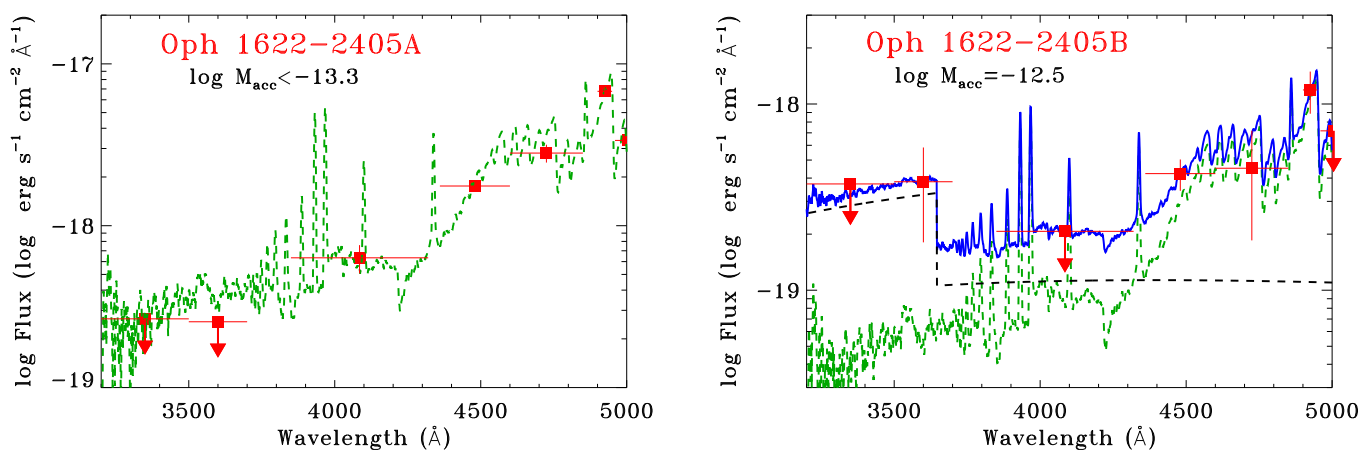

FIGURE 3. Same as Fig. 2. Excess emission is detected from the secondary but not the primary of Oph J162225-240515.

sets the limit on a detectable accretion luminosity. Our very low-resolution continuum spectra from the binned data are sensitive to $26.3 \mathrm{mag}$ at $3600 \AA$ in a 900 s integration. For late-M stars at $140 \mathrm{pc}$, the sensitivity limit of $U \sim 26.3 \mathrm{mag}$. corresponds to an accretion rate of $\sim 10^{-13} M_{\odot} \mathrm{yr}^{-1}$ for a $0.02 M_{\odot}, 0.2 R_{\odot}$ brown dwarf.

\section{ACKNOWLEDGMENTS}

The data were obtained at the W.M. Keck Observatory, which is operated as a scientific partnership among the California Institute of Technology, the University of California and the National Aeronautics and Space Administration. The Observatory was made possible by the generous financial support of the W.M. Keck Foundation.

\section{REFERENCES}

1. Calvet, N., \& Gullbring, E. 1998, ApJ, 509, 802

2. Carpenter, J.M., Mamajek, E.E., Hillenbrand, L.A., \& Meyer, M.R. 2006, ApJ, 651, L49

3. Dahm, S.E., \& Hillenbrand, L.A. 2007, AJ, 133, 2072

4. Gullbring, E., Hartmann, L., Briceno, C., \& Calvet, N. 1998, ApJ, 492, 323

5. Herczeg, G.J., \& Hillenbrand, L.A. 2008, ApJ, 681, 594

6. Kuhi, L.V. 1966, PASP, 78, 430

7. Lodieu, N., Hambly, N.C., Jameson, R.F., \& Hodgkin, S.T. 2008, MNRAS, 383, 1385

8. Luhman, K.L., Stauffer, J.R., Muench, A.A., Rieke, G.H., Lada, E.A., Bouvier, J., \& Lada, C.J. 2003, ApJ, 593, 1093

9. McCarthy, J. K., Cohen, J. G., et al. 1998 SPIE, 3355, 81

10. Mohanty, S., Jayawardhana, R., \& Basri, G. 2005, ApJ, 626, 498

11. Muzerolle, J., Hillenbrand, L., Calvet, N., Briceno, C., \& Hartmann, L. 2003, ApJ, 592, 266

12. Natta, A., Testi, L., \& Randich, S. 2006, A\&A, 452, 245

13. Oke, J.B., Cohen, J.G., Carr, M., Cromer, J., Dingizian, A. \& Harris, F. H. 1995, PASP, 107, 375 O

14. Phillips, A.C., Miller, J., Cowley, D., \& Wallace, V. 2006, SPIE, 6269, 56

15. Scholz, A., et al. 2007, ApJ, 660, 1517

16. Valenti, J.A., Basri, G., \& Johns, C.M. 1993, ApJ, 106, 2024

17. White, R.J., \& Basri, G. 2003, ApJ, 582, 1109 